\title{
Capturing Stillness: Visualisations of dance through motion/performance capture
}

\author{
Ruth Gibson \\ AHRC Creative Fellow Coventry University School of Art \& Design \\ igloo, Unit 301, 449 Bethnal Green Road, London, E2 9QH, United Kingdom \\ http://www.igloo.org.uk \\ ruth@igloo.org.uk
}

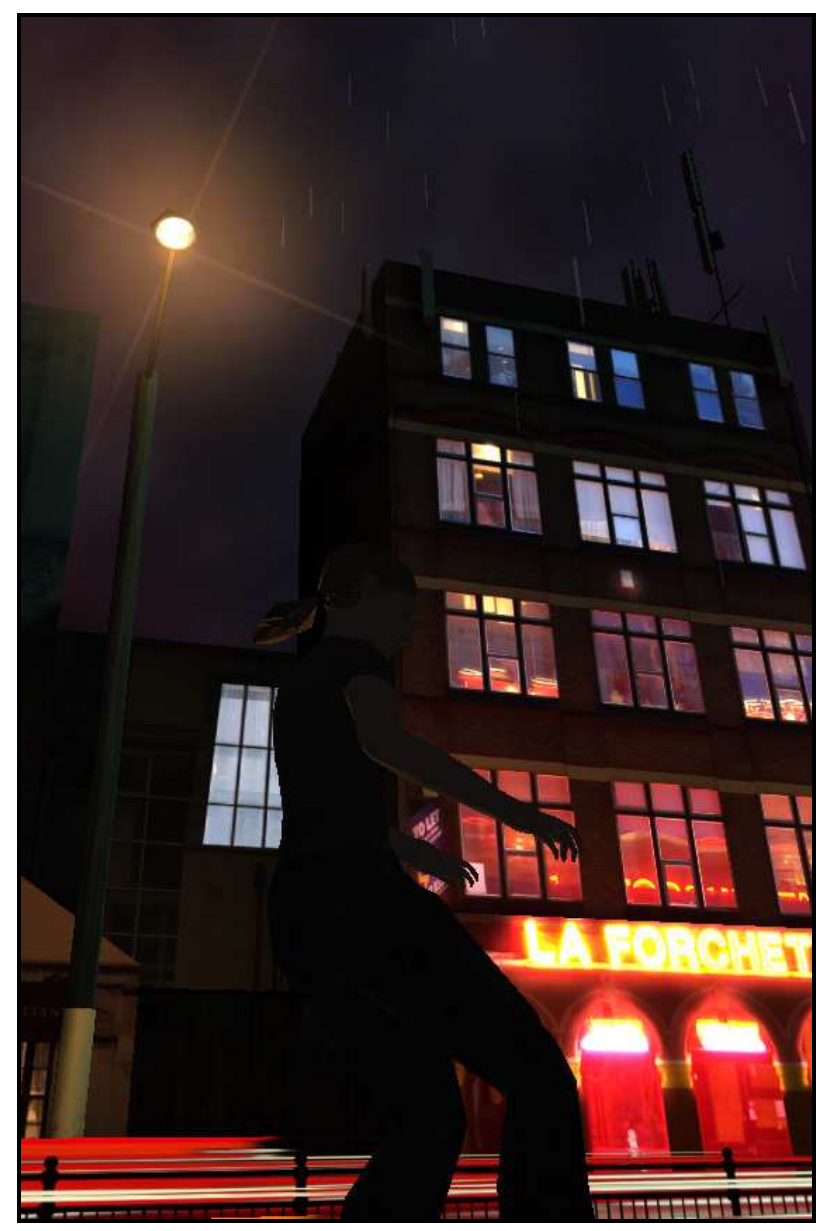

Figure 1: SwanQuake: House 2007

\section{INTRODUCTION}

Dance is increasingly a site of research for experts within the discipline and beyond. With the development of digital technologies, artists and researchers are exploring ways to develop new dance events, to engage with audiences and in doing so, to shed more light on the art form itself whilst expanding its boundaries and limitations. Building on these developments the presentation will share problems and challenges faced when motion capture technology tries to capture dance movement which emerges through somatic movement practices, in particular, an established practice, Skinner Releasing Technique ( SRT).

SRT 'utilises image-guided floor work to ease tension and promote an effortless kind of moving, integrated with alignment of the whole self: Described by Joan Skinner who created the practice as a system of kinaesthetic training that refines the perception and performance of movements, the technique is characterised by attention on the senses, includes concentrated periods of apparent stillness and minimal movement, and movement which is on the floor and is enacted through contact with a partner.

SRT advocates for a kinaesthetic experience, which relates to the scientific notion that we are, ultimately, a psychophysical system of energies: an energy system within the larger energy systems of the universe. SRT is not a generic 'releasing technique' but by employing particular poetic imagery, goes beyond tissues and accesses the subconscious to explore the unexplored in individual movement creation. The imagery cited in the pedagogy alludes to the natural world and spontaneous movement is evoked through sensory imaginings. SRT accesses what is to occur and what has not yet happened and draws upon concepts such as 'constant becoming', 'just being', 'in flux', 'emptiness', 'nothingness' \& 'dynamic stillness'. It is a powerful improvisational technique which taps into different feelings and sensing states facilitating expression, freedom, nuance and the potential for change. The practice promotes the idea that nothing is fixed a creative exploration of technical principles such as multi- directional 
alignment, suppleness, suspension, economy and autonomy.

The presentation will focus on the early stages of a research project, the first of its kind, to visualise SRT through motion capture technology. The principal project's aim is to generate new visualisations based on the capture of SRT that emphasise stillness, interiority and close contact with the floor; to encourage critical engagement with this practice: to expand the possibilities of motion capture technology (hardware and software); to enhance audience kinaesthetic engagement within interactive virtual performance environments. This concentrated study on how the sensing body can interact with motion capture will enable researchers and dancers to understand more about how immersive environments affect user, viewer engagement; and by seeking to reveal more about somatic movement practices, it will enhance kinaesthetic awareness and engagement.

The exploration of the interface between motion capture technology and SRT movement practice opens up a quite different set of questions about the relationship between motion capture/movement tracking and the dancing body. During the presentation examples of 3D interactive artworks with motion captured dance and some of these questions will be referenced.

a) How can the collective experience of SRT movement practice, which generates impulses, dynamics and contact (physical and sensory) between dancers, generate realtime motion capture in relation to pre-recorded motion capture, to mirror the immediacy of the dancers' experience in the studio?

b) Can motion capture 'map' $S R T$ and related practices to test out how notions of embodiment are: 'read', visualised and transmitted; to understand the limits and expansion possibilities of motion capture technology?

c) Can avatar behaviour expose more about stillness and first-person sensorial experience to expand kinaesthetic engagement and awareness?

d) How might the digitally produced bodies for immersive virtual environments provide new viewing experiences?

e) What new experiences for viewers/users can be generated by the development of these visualisations for interactive, immersive virtual environments?

f) What impact do these art events have on the viewer/user's own embodied experience? g) How might the transformation of the dancing body into virtual environments retain or retell something of corporeality?

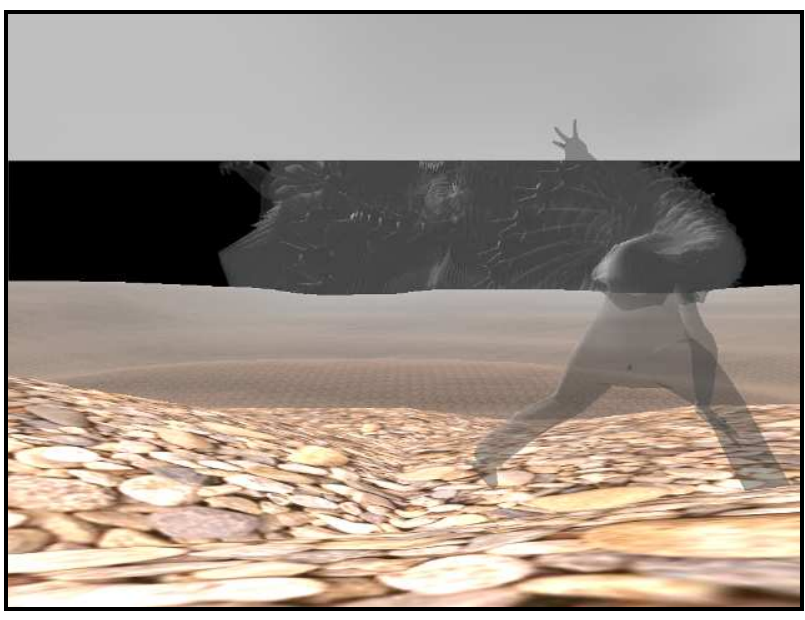

Figure 2: Motion capture glitch animation test - 2006

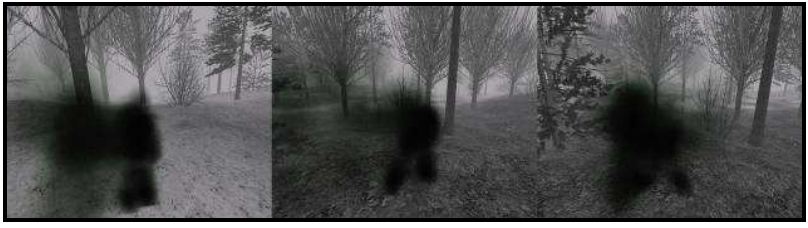

Figure 3: Fog - motion capture character tests 2009

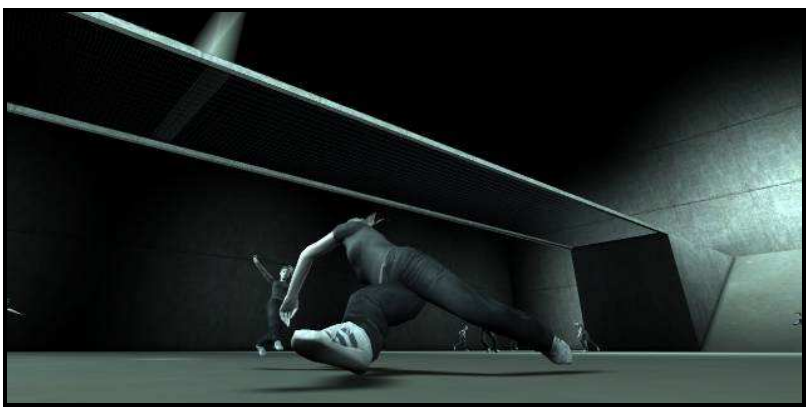

Figure 4: SwanQuake:House - virtual environment with motion captured characters 2007

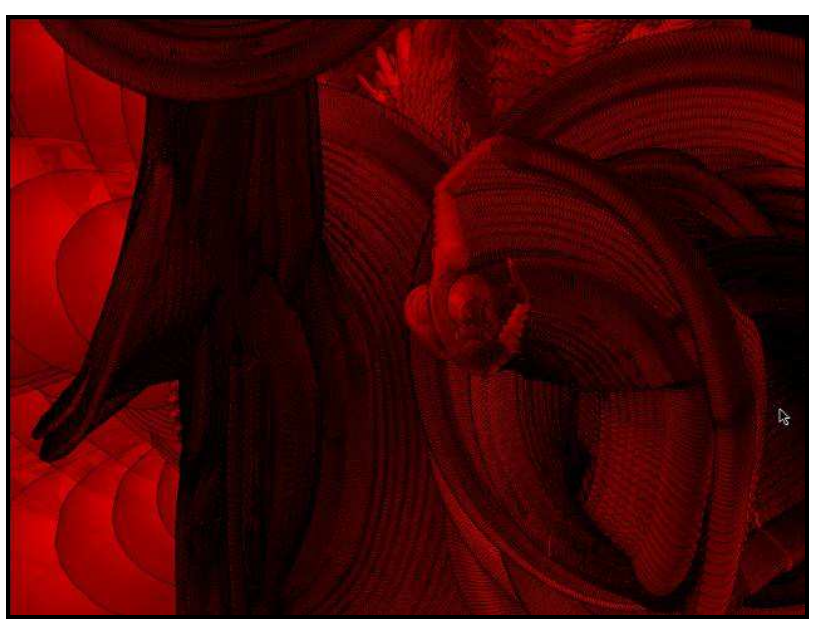

Figure 5: dotdotdot - web-based interactive motion capture animations 2003 


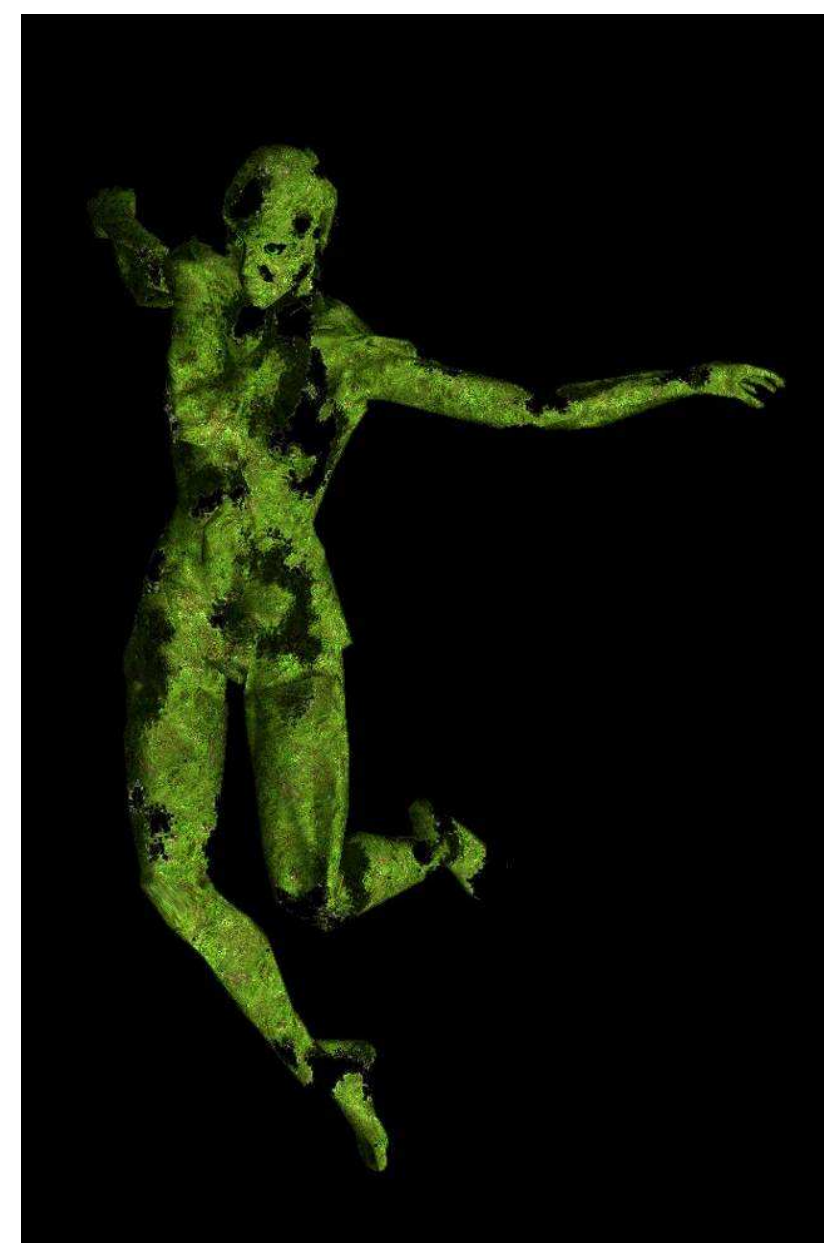

Figure 6: Summerbranch - virtual environment with motion captured characters, character design 2005

\section{REFERENCES}

The poetics of motion capture and visualization techniques: the differences between watching real and virtual dancing bodies: Whatley, S. (Due 2011), Kinesthetic Empathy in Creative and Cultural Practices; Reynolds/Reason (eds), Intellect.

Chapter 3: Jeffries, J. (2009) Blurring the boundaries: Performance,Technology and the Artificial Sublime - An interview with Ruth Gibson and Bruno Martelli, igloo Chatzichristodoulou, Jeffries, Zerihan ( eds) Interfaces of Performance Ashgate. ISBN 9780754675761.

Chapter 4.2: Popat, S. (2011) SwanQuake: House - 'MESSING THE SYSTEM UP' Pitches/Popat (eds), Performance Perspectives: A Critical Reader, Palgrave Macmillian.

Ausdance: Kozel, S. (2004) Connective Tissue: the flesh of the network. Dance rebooted: Initializing the Grid, Deakin University Melbourne Australia, 14 July 2004, Australian Dance Council - Ausdance Inc 2007. ISBN 1875255168.

All images remain the copyright of Gibson/Martelli (igloo). igloo is Ruth Gibson and Bruno Martelli. 